C J Saxon, G Hughes, C Ison, UK LGV Case-Finding Study Group. Health Protection Agency Centre for Infections, London, UK

Background Lymphogranuloma venereum (LGV) infection is now endemic among men who have sex with men (MSM) in the UK. Control of the outbreak has relied on LGV typing and treating of symptomatic patients since previous UK data indicated only $6 \%$ of cases were asymptomatic. However, emerging evidence suggests there may be a significant reservoir of asymptomatic infection.

Methods Twelve UK Genitourinary Medicine clinics that routinely screen all MSMs for Chlamydia trachomatis (CT) at the pharynx, urethra and rectum participated in a case-finding study. All CT-positive specimens in MSMs during the study period (24/09/12 - 07/12/12) were referred for LGV typing and clinical data collected. Descriptive and logistic regression analyses were done.

Results 71 (9\%) LGV and 742 (91\%) non-LGV CT serovars were confirmed. Clinical data were available for 49 (69\%) LGV and 545 (73\%) non-LGV cases. LGV detection was higher in Brighton (10\%), London (10\%) and Manchester (11\%) than in Glasgow (4\%).

$11 / 49(22 \%)$ LGV infections detected were asymptomatic at first presentation; $91 \%(n=10)$ of these were rectal. 3/11 (27\%) LGV infections were 'pre-symptomatic' (asymptomatic at first attendance but symptomatic by the second attendance for treatment). Compared to non-LGV cases, LGV cases were older (median age 39 vs 32 years), more likely to have rectal infection ( $87 \%$ vs $60 \%$; urethral $4 \%$ vs $28 \%$; pharyngeal $4 \%$ vs $23 \%$ ) and to be symptomatic (78\% vs $44 \%)$. In univariate analysis, older age $(\mathrm{OR}=16.9, \mathrm{p}<0.01$; $35-44$ vs 18-24 years), rectal infection $(\mathrm{OR}=5.98, \mathrm{p}<0.0001)$ and symptomatic infection $(\mathrm{OR}=4.20, \mathrm{p}<0.0001)$ were significantly associated with being an LGV case.

Discussion Asymptomatic rectal LGV is considerably more common than previously reported in the UK. This could reflect changing screening practises for MSM, or evolving LGV epidemiology. LGV typing in asymptomatic rectal CT may be warranted to reduce ongoing transmission. The effectiveness of current CT treatment regimens in asymptomatic LGV needs assessment

\section{P3.139 EARLY INCUBATING GONORRHOEA AND CHLAMYDIA INFECTIONS IN MSIM WITH AN INDICATION FOR HIV POST EXPOSURE PROPHYLAXIS (PEP)}

doi:10.1136/sextrans-2013-051184.0598

${ }^{1} \mathrm{~N}$ H N de Vrieze, 'M van Rooijen, ${ }^{1,2} \mathrm{H}$ J C de Vries. ${ }^{1}$ Public Health Service, Cluster of Infectious diseases, Amsterdam, The Netherlands; '2Academic Medical Centre, Department of Dermatology, Amsterdam, The Netherlands

Introduction A PEP indication is an ideal opportunity for safe sex promotion and STI screening. Since 2010 the STI outpatient clinic in Amsterdam, the Netherlands, offers PEP to HIV negative men who have sex with men (MSM) who had unprotected receptive anal intercourse within the last 72 hours. If STI screening is performed at the moment of PEP request, early incubating chlamydia and gonorrhoea infections acquired during the unsafe sex act, are possibly missed. We aimed to determine if chlamydia and gonorrhoea screening should be repeated in MSM 2 weeks after a PEP indication.

Methods We included all MSM visiting the STI clinic with a PEP request in the period from April 2010 until December 2012. STI testing was offered to all MSM during the PEP evaluation visit. Men were screened for urethral, anal and pharyngeal infections based on their practised sex techniques. If PEP was indicated a visit was planned 2 weeks later to repeat gonorrhoea, and chlamydia screening.

Results 447 consultations MSM requested PEP and in 325 (72.7\%) PEP was indicated. In 50/325 (15\%) cases at least one STI was diagnosed at the moment of PEP indication. 172 (52.9\%) cases returned after 2 weeks of whom in $9(5.2 \%)$ cases at least one previously undiagnosed infection was found ( 3 rectal chlamydia, 3 rectal gon- orrhoea, 2 rectal chlamydia/gonorrhoea double infections, and 1 pharyngeal chlamydia).

Conclusion Repeated chlamydia and gonorrhoea screening 2 weeks after a PEP indication in MSM revealed 5.2\% additional, possibly early incubating, infections. Yet, $47.1 \%$ of MSM did not show up for the second screenings visit. Therefore STI screening should be offered at the PEP indication visit and preferably repeated after 2 weeks to exclude early incubating chlamydia and gonorrhoea infections.

\section{P3.140 LYMPHOGRANULOMA VENEREUM AMONG MEN WHO HAVE SEX WITH MEN IN THE NETHERLANDS: AN UPDATE ON THE CURRENT SITUATION}

doi:10.1136/sextrans-2013-051184.0599

${ }^{1} \mathrm{~N}$ E Koper, ${ }^{1,2} \mathrm{M}$ A B van der Sande, ${ }^{3} \mathrm{H}$ M Götz, ${ }^{1} \mathbf{F}$ D H Koedijk, ${ }^{4} \mathrm{On}$ behalf of the Dutch STI clinics. 'National Institute of Public Health and the Environment, Bilthoven, The Netherlands; ' 2 Julius Center for Health Sciences and Primary Care, University Medical Centre Utrecht, Utrecht, The Netherlands, ${ }^{3}$ Public Health Service Rotterdam Rijnmond, Rotterdam, The Netherlands; ${ }^{4}$ Dutch STI clinics: A van Daal (East), AP van Leeuwen (North-Holland Flevoland), F de Groot (North), CJPA Hoebe (Limburg), M Langevoort (Utrecht), AM van Camerijk (South-Holland North), J van de Sande (Zeeland-Brabant), $\checkmark$ Wieërs (South-Holland South), Bilthoven, The Netherlands

Background Since 2003, an epidemic of Lymphogranuloma venereum (LGV) among men who have sex with men (MSM) in Europe has been on-going. This study determined how the epidemic of LGV in MSM visiting STI clinics in the Netherlands has evolved since 2006. Methods Data on MSM from the national STI surveillance system for 2006-2011 were analysed. LGV testing and positivity rates were calculated and multivariable logistic regression analysis was applied to study risk factors for LGV. In addition, data on the number of cases and positivity rate for the first six months of 2012 were analysed.

Results Between 2006 and 2011, 75\% of cases of anorectal chlamydia in MSM were tested for LGV, but this varied between regions from $7 \%$ to $97 \%$. In this period, $414 \mathrm{LGV}$ cases were diagnosed, with a mean positivity rate of $8.7 \%$. Positivity rate decreased from $14 \%$ in 2007 to $6 \%$ in 2011 . However, results from the first half year of 2012 showed that positivity rate increased sharply to $14.7 \%$ ( $n=106$ new cases). In multivariable analyses on all cases, risk factors for LGV were HIV positivity (OR $=4.1$; 95\% CI: 3.2-5.3), STI symptoms $(\mathrm{OR}=4.1 ; 95 \% \mathrm{CI}: 3.1-5.4),>50$ sex partners in the past 6 months $(\mathrm{OR}=3.7 ; 95 \% \mathrm{CI}: 1.1-12.4)$, older age (40-44: $\mathrm{OR}=2.1 ; 95 \% \mathrm{CI}$ : 1.5-2.8), no condom use with last partner ( $\mathrm{OR}=2.2 ; 95 \% \mathrm{CI}: 1.2$ 3.9 ) and exclusively having sex with men (OR $=2.2 ; 95 \% \mathrm{CI}: 1.1$ 4.2), No significant changes over time were found in risk behaviour. Conclusions Since 2006, the annual incidence for LGV fluctuated but was consistently higher than before 2003 and is increasing sharply in the first half of 2012. Regional differences in LGV testing rates limit nationwide LGV surveillance, leading to an underestimation of the real LGV incidence. Characteristics of MSM affected by LGV did not change over time, so prevention strategies and timely and adequate diagnostic towards this specific high-risk group should be intensified.

\section{P3.141 CORE WITHIN A CORE? ANALYSIS OF LGV PATIENTS WITH A KNOWN REPEAT INFECTION}

doi:10.1136/sextrans-2013-051184.0600

'M Rönn, ${ }^{2} \mathrm{G}$ Hughes, ${ }^{1,2} \mathrm{P}$ White, ${ }^{2} \mathrm{~S}$ Simms, ${ }^{3} \mathrm{C}$ Ison, ${ }^{1} \mathrm{H}$ Ward. ${ }^{1} / \mathrm{mperial}$ College London, London, UK; ${ }^{2}$ Health Protection Agency, London, UK; ${ }^{3}$ Sexually Transmitted Bacteria Reference Laboratory, Health Protection Agency, London, UK

Background Lymphogranuloma venereum (LGV) is a re-emergent STI that particularly affects HIV-positive men who have sex with men. During enhanced surveillance in the United Kingdom a number of individuals presented with LGV re-infection. Determining 